\title{
Visual Complexity Analysis Using Taxonomic Diagrams of Figures and Backgrounds in Japanese Residential Streetscapes
}

\author{
G. M. W. L. Gunawardena, Yoichi Kubota, and Kiyotaka Fukahori \\ Course of Environmental Science and Infrastructure Engineering, Saitama University, 255 Shimo-Okubo, Sakura-ku, \\ Saitama-shi, Saitama-ken 338-8570, Japan
}

Correspondence should be addressed to G. M. W. L. Gunawardena; w.lakpriya@gmail.com

Received 8 May 2015; Revised 26 August 2015; Accepted 7 September 2015

Academic Editor: Eric Koomen

Copyright (C) 2015 G. M. W. L. Gunawardena et al. This is an open access article distributed under the Creative Commons Attribution License, which permits unrestricted use, distribution, and reproduction in any medium, provided the original work is properly cited.

\begin{abstract}
This study was conducted with the objective of finding the perceivable effect of figures and backgrounds on residential streetscapes and their connections in visual complexity. The visual complexity depends on the extent of information a viewer can observe from a visible area. The information includes a number of visual elements along the streetscapes and their diversity and interconnections. The aim of this research was to analyze the structural hierarchical visual complexity of the streetscapes, caused by varied spatial arrangement and numerous spatial connections of the perceivable visual elements. The visual elements of 60 residential streetscapes were classified into figures and backgrounds using human perception of 20 subjects. The identified figures and backgrounds were arranged in a taxonomic diagram representing their connections. These taxonomic diagrams reflect the structural hierarchical visual complexity. Finally, taxonomic entropy was applied to statistically analyze the structural hierarchical visual complexity. When the taxonomic diagram is vertically and horizontally lengthy and the arrangement of the elements of taxonomic diagram is irregular, the complexity increases depicting a large number of figures whose spatial connections impart a high visual complexity to the streetscapes.
\end{abstract}

\section{Introduction}

Streets are important elements of a city, and residential streetscapes especially are important places to perceive and appreciate the quality of life of the residents. A street is a narrow and linear urban space lined by buildings, used for movement and other activities. The street, frontage of residential buildings along the street such as commercial sign boards, skyline, street pavement, lighting, vegetation, are all important parts of a street's visual elements. These visual elements show a hierarchical order of connections and they are cumulatively responsible for the chaotic nature of the streetscapes. This chaotic nature of streetscapes has been measured using two definitions: visual diversity and visual complexity. Visual diversity explains the variety aspect of visual elements while visual complexity explains both connections and the variety aspects of visual elements. Thus, measuring visual complexity is more important than the visual diversity since it measures both variety and connections of the visual elements. This complexity is called structural hierarchical visual complexity. Although there are a number of techniques to measure visual complexity in literature such as Shannon's entropy, fractal dimension analysis, and saliency, they measure only one aspect of visual complexity, which is variety. Therefore, these available methods do not represent the actual visual complexity on landscapes. Detailed explanations about available methods are given in a separate section in this paper. Due to this reason, the introduction of a method to measure structural hierarchical visual complexity representing both aspects of visual complexity is a timely requirement. In this research an attempt was undertaken to create a method to give a numerical value to visual complexity considering both the variety and connection aspect of visual complexity in a hierarchical manner. Thus, the objective of this study is to develop an objective analysis method which represents both variety and the connections aspects of visual complexity on streetscapes. Since the forward and backward streetscape 
views were analyzed for structural hierarchical visual complexity, a comparison of the forward and backward analysis results was undertaken as a specific objective to identify any difference between forward and backward structural hierarchical visual complexities. The hypothesis of this study is that when the spatial arrangement (variety) and the invisible connections (dependency) of the figures and backgrounds along streetscape become complex, the perceivable visual extent increases thus intensifying the structural hierarchical visual complexity. Therefore, structural hierarchical visual complexity can be measured by analyzing the taxonomic diagrams which represent the variety and dependency of perceivable visual elements along streetscapes. A detailed explanation about taxonomic diagrams is given in a separate section in this paper. To draw and analyze the taxonomic diagrams, two approaches were applied: Gestalt theory $[1,2]$ of visual perception and the taxonomic entropy [3]. The use of these techniques is explained in detail under separate sections.

\section{Visual Complexity}

Visual complexity is traditionally defined as the level of features contained within a place or an image. In environmental psychology, complexity is related to the involvement component, which translates into enquiring "how much there is to see in a visual array?," and to the concept of affordance that refers to what a perceived scene has to offer as far as the perceiver is concerned [4]. Complexity is broadly of four categories: (i) structural complexity, based on spatial dimensions of objects; (ii) functional complexity, based on temporal dimension of objects; (iii) structural hierarchical complexity, based on spatial scale of the objects, and (iv) functional hierarchical complexity, based on temporal scale of the objects [5,6]. Reference [7] contends that the complexity of a pattern increases with increasing number of independently selected elements it contains. Thus, complexity can be defined in many ways, and measuring complexity is important for the identity of a landscape and for maintaining appropriate complexity among visual elements to ensure proper balance between nature and the manmade landscapes.

In this study, structural hierarchical visual complexity is the main concern and it can be defined as the amount of information a perceiver could perceive from the visibility area of a streetscape. Information includes number of visual elements, diversity, and the invisible connections among them to sense a complexity to the perceiver. Density, pedestrian friendly design, and diversity of a streetscape are included in the structural hierarchical visual complexity. It represents the invisible structure associated with visual elements on landscapes.

2.1. Visual Complexity Analysis from Literature. In streetscapes, the interest and preference of pedestrians are shown to be heavily dependent on the perceived complexity [8]. Specifically, pedestrians are prone to prefer streets perceived to be of high complexity. Streetscape complexity is also found to influence driving behavior and performance [6]. Therefore, proper measurement and quantification of visual complexity are beneficial for urban life. There are many methods to measure visual complexity. This chapter presents an overview of the past researches on visual complexity measurement.

Fractal dimension analysis is one popular objective analysis method to measure visual complexity. This method describes how patterns of different magnifications combine to form fractal shape [9]. Reference [9] argues that most of the "natural" world and much of the built environment in combination are inherently irregular and cannot be properly described using the concepts of only one, two, or three dimensions, Euclidean geometry. Reference [9] derived the term "fractal" from the Latin verb frangere, "to break," and the adjective fractus, meaning irregular and fragmented, and used the term to describe objects that were not necessarily just irregular but demonstrated repeating patterns when examined at increasingly smaller scales that demonstrated "scale invariance" or self-similarity. It is this appearance of self-similarity that is quantified by the concept of fractal dimension. All fractal objects have in common the notion of fractal dimension: this enables the degree of irregularity of an object or pattern to be measured and represented as a number. The fractal dimension is represented by $D$ and lies between the Euclidean dimensions of 1, 2, or 3. For example, the fractal dimension of an irregular line representing a coastline would lie between 1 and 2: it is not a simple straight line, which would have only one dimension, but it is also not a fully two-dimensional plane. The fractal dimension lies between the two and is represented as a noninteger number, whereas Euclidean dimensions are integers. Essentially, fractal dimension is a measure of how well a particular object fills the space in which it is drawn. When the complexity and richness of the repetitive structure increase, its fractal dimension value moves closer to two [9]. Thus, the value of fractal dimension changes with varying complexity. However, fractal dimension measures only one aspect of visual complexity, that is, variety. There is no indication on the invisible connections among the visual elements and the complexity occurring due to the invisible structure of visual elements of landscapes in this analysis method. Therefore, fractal dimensions do not represent the structural hierarchical visual complexity which is the main focus of this study.

Another objective way of measuring complexity is using spatial frequency. In this method, the amplitude of highfrequency components were used for recognizing complex objects [10]. Similarly, specific relationships among frequency components in the phase spectrum are crucial for visual recognition of complex scenes [11]. Based on the characteristics of spatial frequency, [12] derives a complexity measure defined as the product between the effective image area and median frequency of the Fourier spectrum. Reference [13] uses the components of this measure to analyze the complexity in landscapes. This method also addressed only one aspect of complexity, which is variety. In this method, the spatial frequency which comes from different types of objects was analyzed to identify the visual complexity and it has no measurement for the other important aspect (connection) of visual complexity.

It has also been shown that the presence of image edges is related to visual complexity. This inspired a simple and 
efficient measure known as perimeter detection [14]. This objective measurement consists in counting the number of pixels that form image edges. This procedure can be easily applied to real-world scenes by using edge-detection algorithms [14]. This method does not take into account the connections aspect of visual complexity.

Reference [3] proposes an objective framework, called feature congestion, to measure visual clutter, an attribute closely related to complexity. Within this framework, several image characteristics, such as contrast, color, and orientation, are combined into vector space. Clutter is then determined by the covariance of the space, calculated at each location of the image. This method also addresses only the variety aspect of visual complexity.

Another method of measuring visual complexity is according to the definition of information theory [15]. An example of information-based measure is the size in bytes of the digital image file, created according to coding standards, such as JPEG and TIFF. Theoretically, the file size should increase as the amount of information increases [16].

Some researchers applied statistical approaches, such as Shannon's entropy, to measure visual complexity [3] while reference [17] used a manual approach to segment meaningful elements of street houses such as windows, doorways, and overall volumes of facades. Complexity was then measured, based on the number and variety of those elements. Reference [18] used a manual technique to segment street skylines and then fractal dimension to assess the complexity of the skylines. These methods also deal with only the variety aspect of visual complexity.

Thus, the visual complexity analysis methods which were found in the past literature addressed only the variety aspect of complexity and it is called structural complexity. Structural complexity is more similar with the diversity measurement.

2.2. The Research Strategy. As stated above, there are many methods in literature to measure visual complexity; some of them are statistical approaches and some are manual approaches. Most of them measure complexity, based on the number or the appearance of the visual elements which is the variety aspect of visual complexity and it is called structural visual complexity. It is similar to the visual diversity. The method proposed here is different from the existing ones in two ways: first, this study measures structural hierarchical visual complexity, not the simple structural visual complexity, which was a measure, most researchers used; second, in measuring the structural hierarchical visual complexity, two technique, namely, Gestalt figure and background concept $[1,2]$ and the taxonomic entropy [3], were applied. The spatial arrangement and the spatial connections of visual elements along the streetscape are responsible for the structural hierarchical visual complexity. Thus, it addressed both essential aspects of visual complexity and explains the real visual complexity of streetscapes than just simple structural complexity. Visual elements could be classified into figures and backgrounds, based on the Gestalt theory. One visual element may contain more than one figure or background. Using cues of differentiating figures and backgrounds such as size, shape, orientation, color, and distance, the perceivable figures and backgrounds in main visual elements can be identified. The invisible sequence of arrangement of these figures and backgrounds and their invisible mutual connections can be visualized through taxonomic diagrams. The taxonomic diagrams represent the structural hierarchical visual complexity. This is a novel approach for measuring visual complexity. When the spatial arrangement and the spatial connections of the figures and backgrounds along streetscape become complex, the visual complexity increases; therefore, visual complexity can be measured by analyzing the taxonomic diagrams of figures and backgrounds. For this analysis, taxonomic entropy was applied. Thus, for this research an attempt was made to introduce a method for measuring structural hierarchical visual complexity by using Gestalt's explanation on the figure and background and the taxonomic entropy.

\subsection{The Concept of Figure and Background. Elements are} perceived either as figures (distinct elements of focus) or as background (the background or landscape on which the figures rest). It is a type of perceptual grouping, which is vital to recognizing objects through vision [19]. Identifying an element, as a figure or as a background, is based on many factors of probabilistic nature; they are the size, shape, movement, color, edge assignment, and the distance of elements. Any visual element can be grouped into figures or backgrounds. One element to which a viewer pays attention provides additional visual perception on the detail of the element and if viewer focuses it, differentiation process of figure and background may be repeated. This focusing process of visual interest can be defined as hierarchical differentiation mechanism of figure and background. When a visual element has several figures and backgrounds, they can be arranged in a hierarchical order to show the invisible connections among them. Thus, the identified figures and backgrounds could be arranged in taxonomic diagrams to display the variety and the invisible connections among them.

\section{Materials and Methods}

3.1. Study Area. The research was conducted in the urban residential areas around the Saitama University in Japan. Figure 1 shows streetscapes in the study area.

For the research, 60 urban residential streetscapes were selected from the vicinity of the Saitama University. Each streetscape was around $200 \mathrm{~m}$ in length. The streetscapes were selected from the urbanization controlled areas, medium urbanized areas, and highly urbanized areas according to the information obtained from Japanese land use classification maps. The selected streetscapes had approximately similar plot sizes. Twenty streetscapes each from urbanization controlled areas, highly urbanized areas, and medium urbanized areas were selected for further analysis. All streetscapes were selected from the residential areas. Majority of residential buildings were two-story buildings. Occasionally single story and three-, or more, story buildings were located along the streetscapes. Retail shops, laundries, and small scale business places could be observed in some streetscapes as 

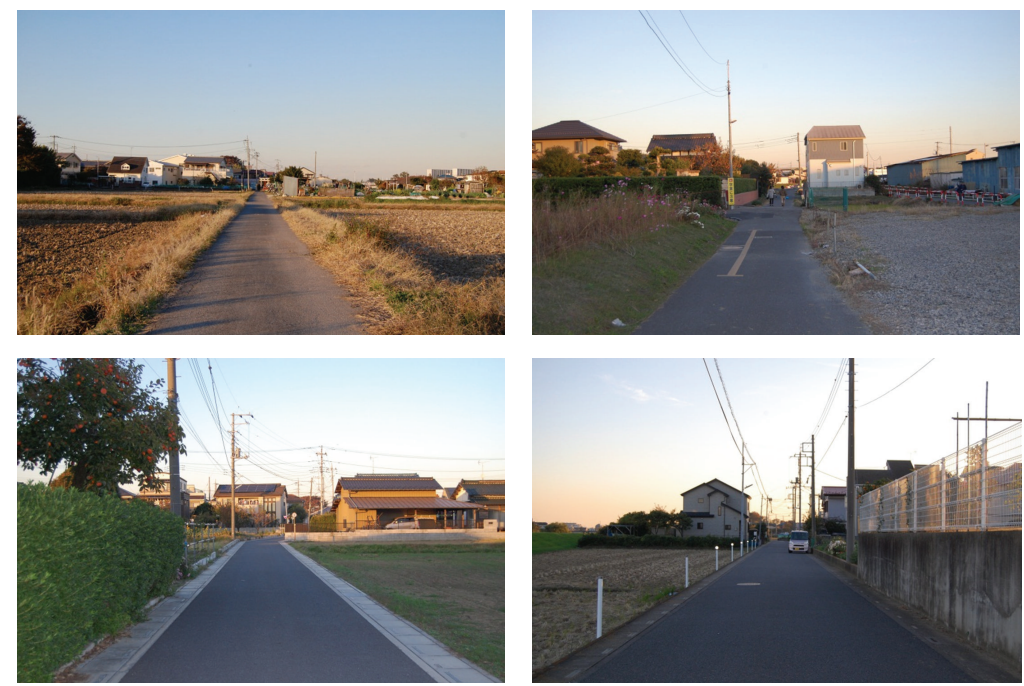

(a) Sample streetscapes from urbanization controlled areas
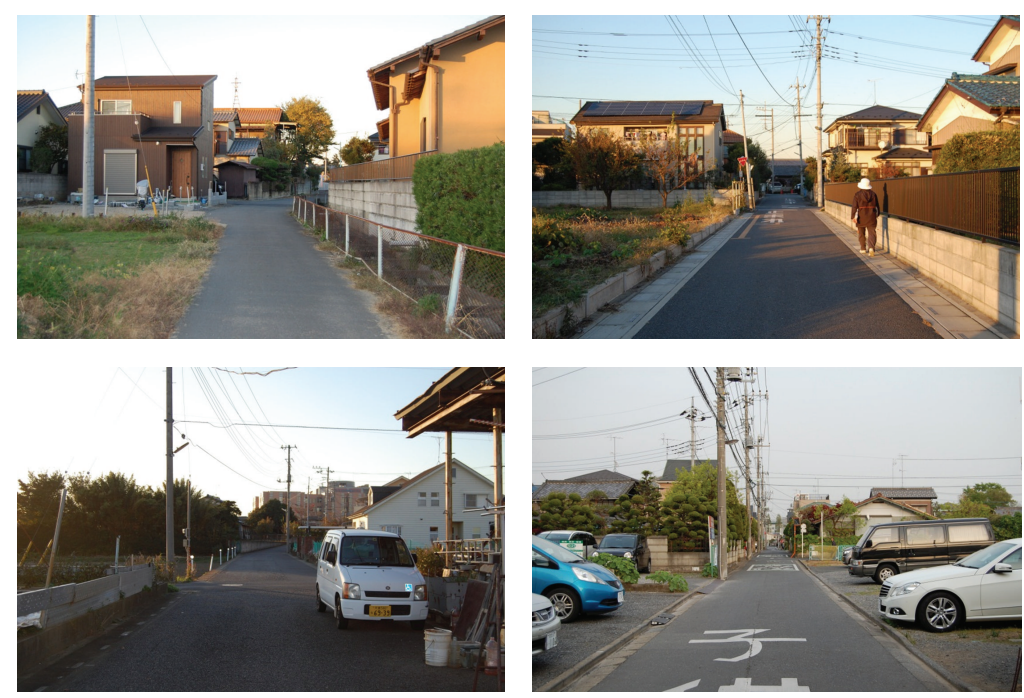

(b) Sample streetscapes from medium urbanized areas
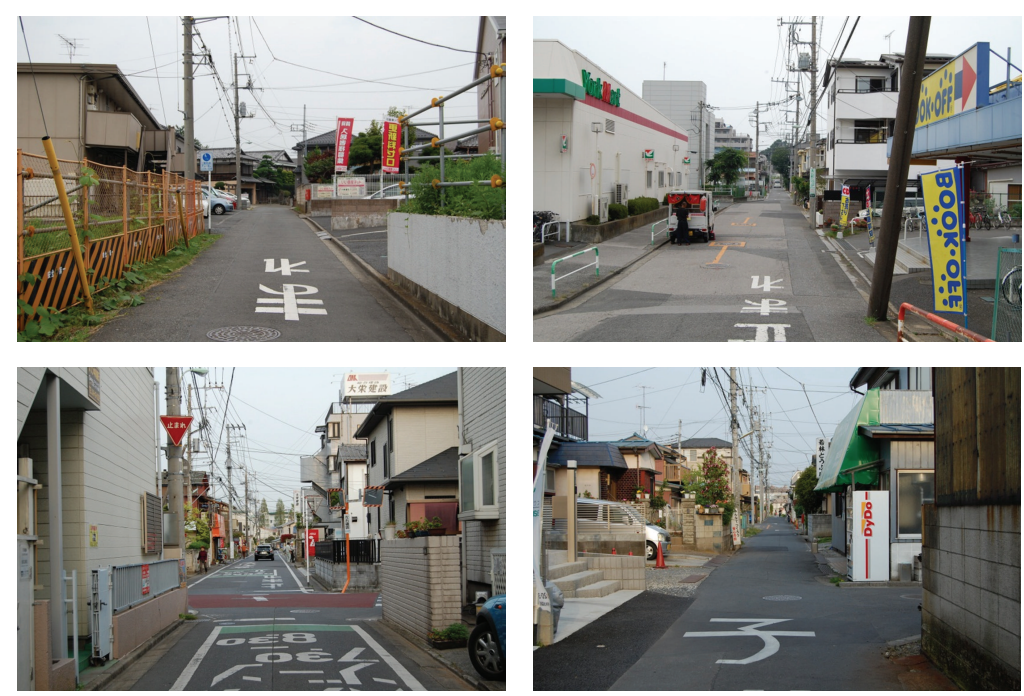

(c) Sample streetscape from highly urbanized areas

Figure 1: The study area. 
TABLE 1: Information about the subjects who participated in the survey.

\begin{tabular}{lccc}
\hline Number of subjects & Gender & Age & Nationality \\
\hline 5 & 3 females and 2 males & $25-30$ & Japan \\
5 & 3 females and 2 males & $25-35$ & Sri Lanka \\
3 & 2 males and 1 female & $25-30$ & Pakistan \\
3 & 2 females and 1 male & $25-30$ & Nepal \\
2 & 1 male and 1 female & $25-30$ & Bangladesh \\
2 & 2 females & $25-30$ & Myanmar \\
\hline
\end{tabular}

well. Vending machines were located on either side of street frequently.

3.2. Analysis of Perspective Views. The perspective views of the selected streetscapes were analyzed with the help of 20 subjects of different nationalities from the Saitama University. All the subjects were students at Saitama University either doctoral degree students and masters students. None of them were selected from the urban study stream. Table 1 displays the information about the subjects who participated in the survey.

The residential streetscapes were presented as photographs taken from five viewpoints and shown on computer screens to the subjects. The author personally stayed with each subject until they completed the survey to help them when there were any unclear situations. The views were displayed individually to each subject and approximately three hours was required to complete the survey with each subject. Each subject was asked to select one to five major visual elements from each viewpoint in the first round. Those are the first eye-catching elements from each viewpoint. Then, each main visual element was further classified into subfigures and subbackgrounds by each subject to identify the sequential vision of the subelements of the main visual element. This procedure was undertaken to identify the most eye catching main visual elements and their subelements which are responsible for the structural hierarchical visual complexity of a main visual element. This is essential to create the taxonomic diagrams showing variety and the invisible connections among figures and backgrounds of main visual elements to measure the structural hierarchical visual complexity of each viewpoint. The same procedure was undertaken for the 60 selected streetscapes.

3.3. The Data Arrangement. The subjects identified figures and backgrounds based on the cues of perception, size, shape, color, edge assignment, and the distance of elements. Usually subjects explained about the figure elements standing against monotonous background. The detailed explanations of figures and backgrounds, given by 20 subjects, were analyzed individually, based on the knowledge of the streetscapes. A major visual element may contain more than one figure and a background. After classifying the figures and backgrounds for each major visual element of the 20 subjects, the probabilities of highlighting visual elements as figures or backgrounds were calculated. Usually, the subjects explained about the figure element, since they are the eye-catching elements in the view. Therefore, the probability of mentioning an object as a figure was considered to identify the figures and backgrounds in the view. If the probability of an element being classified as a figure was higher than $75 \%$, then that visual element was classified as a figure element, and if it is less than $75 \%$, then the element was classified as a background element. With the help of probability values, the figures and the backgrounds were classified and arranged as taxonomic diagrams for statistical analysis. A sample of the taxonomic diagram of figures and backgrounds, created for a major visual element, is given in Figure 2. Labels with red letters are figures and those with black letters are the corresponding backgrounds.

3.4. Data Analysis. The data was analyzed to determine the structural hierarchical visual complexity of each residential streetscape. For this analysis, taxonomic entropy [20] was applied.

Taxonomic indices are introduced by ecologists by incorporating information about the degree of ecological similarity among species in a community [20]. A community composed of species that are distantly related taxonomically or have diverse ecological roles and characteristics is intuitively more diverse than a community composed of similar or near-similar species. Therefore valuable indices of diversity should account for differences related to functional type or morphology, taxonomic relatedness amongst species, or genetic distances, because ecological differences between taxa are believed to be reflected in each of them [11]. Because taxonomic relatedness is most commonly used to determine the dissimilarity or distance between species, these indices of diversity are generally referred to as taxonomic diversity indices [21].

The concept applied for this research is similar to the taxonomic relatedness concept. If a main visual element has many subvisual elements (variety) with connections (dependency), then the structural hierarchical visual complexity of the main visual element becomes high. This similarity can be further explained using an example.

Example. Visualize a pond consisting of different types of fish species, each taxonomically different from the other. The biodiversity of the pond is measured by using the taxonomic diagram of these fish species. Similarly, a house in a streetscape is a pool of subvisual elements with invisible connections. The structural hierarchical visual complexity of the house can be measured by using the taxonomic diagram of the variety and the dependency. By way of further elaboration, the taxonomic diagrams of these two examples are given in Figures 3 and 4.

The taxonomic diversity is calculated by using the probability of different types of species and the total distance between the species and their great grandmother. Hence, for diversity calculation, only the probabilities and the distances were applied. Other information is immaterial for the calculation.

Similarly, for structural hierarchical visual complexity analysis by using taxonomic diagrams, the probabilities of each type of branches in the taxonomic diagram and the total 

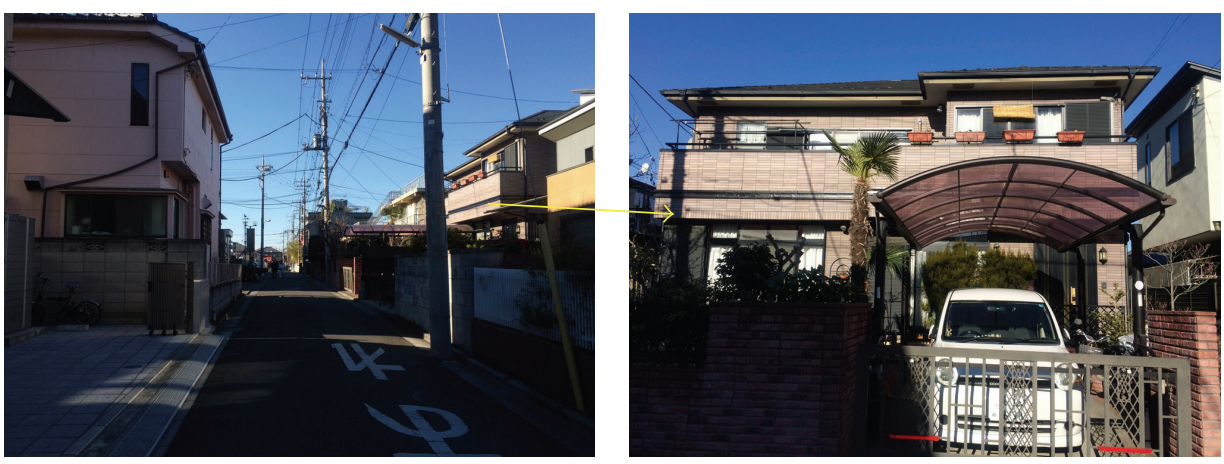

(a) A main visual element selected by subjects

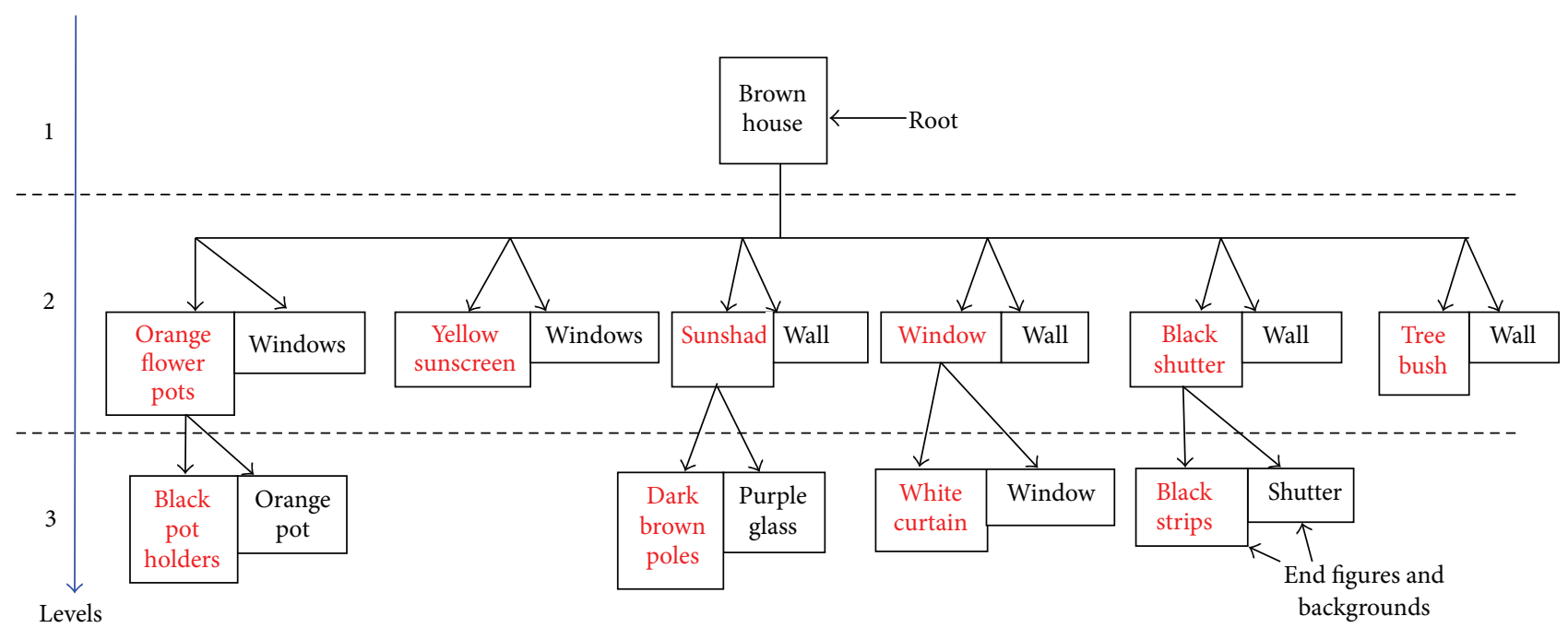

(b) Taxonomic diagram drawn to the main visual element

FIgURE 2: A sample of (a) a main visual element selected by subjects and (b) taxonomic diagram drawn to the main visual element.

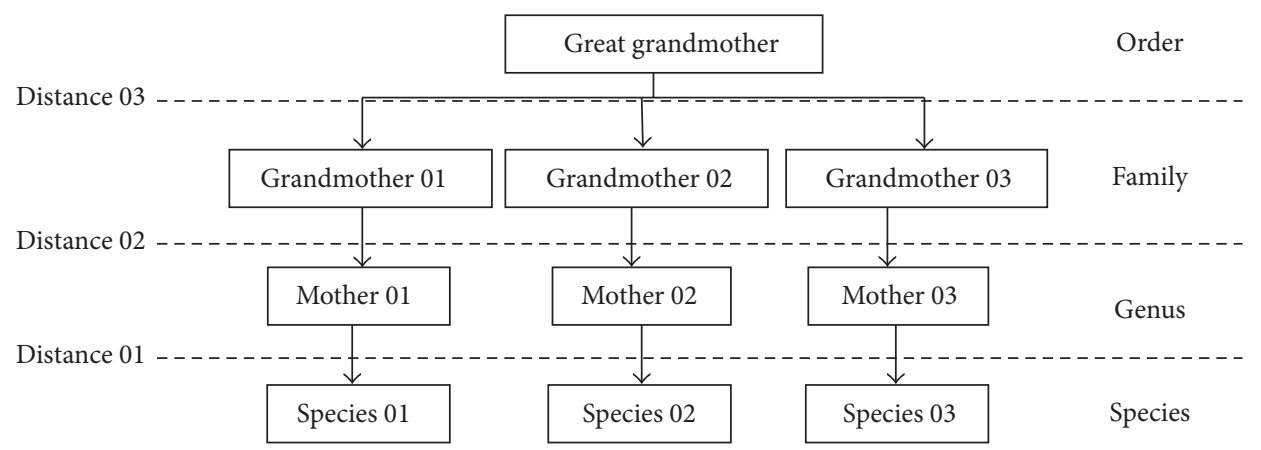

FIGURE 3: The taxonomic diagram of biodiversity.

distance from the end figure to the main visual element were taken into account. In biodiversity assessment, the probability of the species is taken into account, and, in structural hierarchical visual complexity analysis, the probability of each type of branch is taken into account. Besides, in visual complexity analysis, the same end visual elements will not reappear as probabilities as in biodiversity assessment and the biodiversity concept solely does not apply to visual complexity analysis. Therefore, different types of branches were considered as one indicator of the structural hierarchical visual complexity.

The taxonomic entropy index was selected for the data analysis of the study:

$$
H=-\sum p_{i} \log _{2} k_{i}
$$

where $H$ is taxonomic entropy, $p_{i}$ the probability of $i$ th species, and $k_{i}$ the taxonomic distinctness of $i$ th species. 


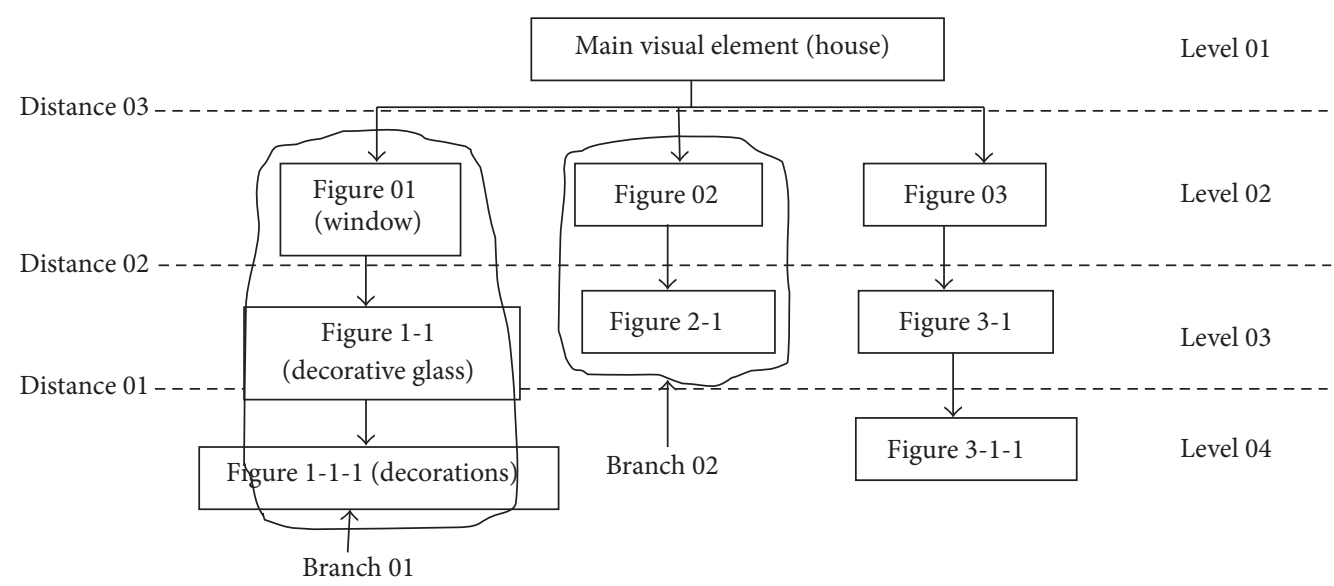

FIGURE 4: The taxonomic diagram of visual complexity.

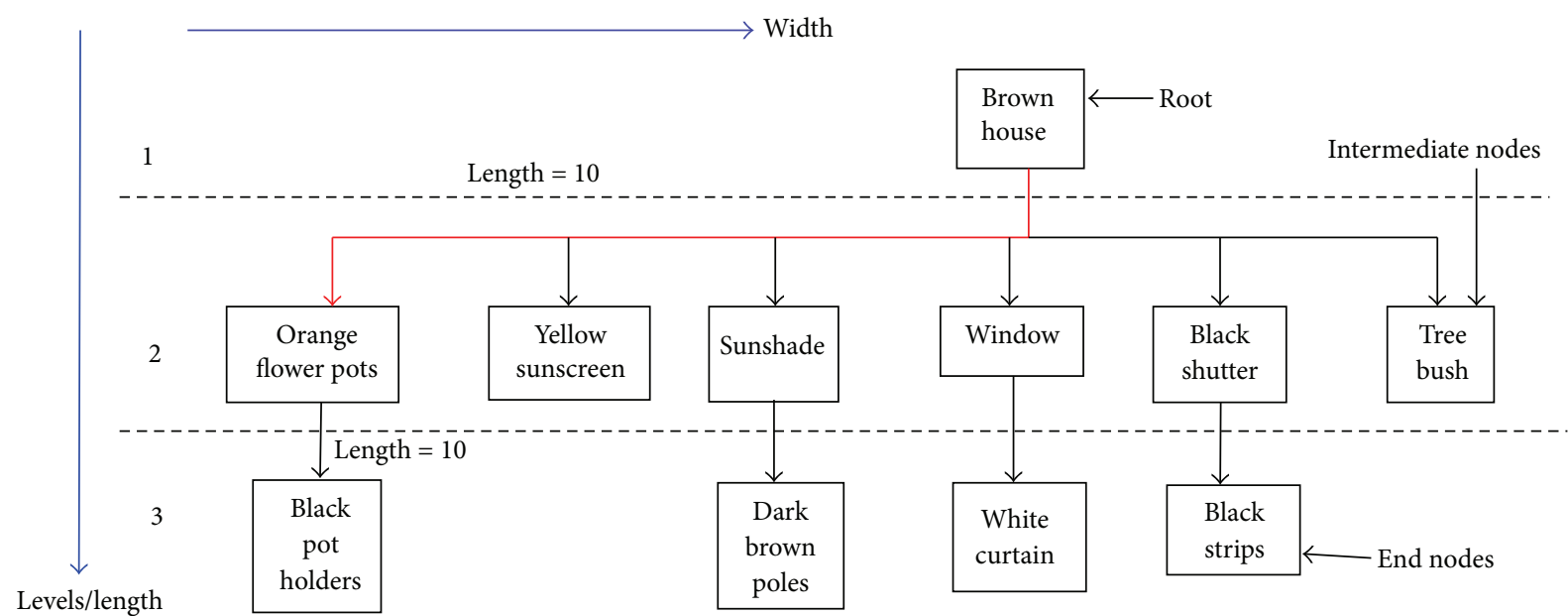

FIGURE 5: Streetscape taxonomic diagram.

For this research, the original equation was slightly modified to match the objective of present research. Figure 5 shows the measurement taken from the streetscape taxonomic diagrams for index calculation. In streetscape taxonomic diagrams two features are available, figures which are similar to the species in biodiversity, and the backgrounds, which are similar to the habitat in biodiversity. Since figures are the most important contributor for the streetscape visual complexity, they were selected for the taxonomic entropy calculation of the streetscape.

Taxonomic diagram has different types of branches, such as two-level and three-level branches. The complexity of taxonomic diagram varies, depending on the number and length of the branches. The number and length of branches differ based on the number of visual elements explained by the subjects; accordingly, the complexity was computed using the number of branches and their total length:

$$
H=\sum p_{i} \log _{2} k_{i}
$$

where $p_{i}$ refers to the probability of $i$ th branch and $k_{i}$ to its total length. For length calculation, value 10 was given to the length between two levels to avoid errors in applying logarithmic values in the equation. Thus, the total length of a branch with two levels is 10 and that of a branch with three levels 20. Because the logarithmic values are nonnegative, it is not necessary to get the negative sum as explained in the original equation.

\section{Results and Discussion}

The residential streetscapes were classified into three groups, streetscapes in urbanization controlled areas, streetscapes in the highly urbanized areas, and the streetscapes in medium urbanized areas, based on the information obtained from the land use maps of Saitama city. The subjects tended to select the objects from the perspective views, based on the cues explained by Gestalt: the size, shape, color, edge assignment, and the distance of objects. Therefore, the major selections for further classification were the houses visible near the street line, the sign boards, street signs, retail shops, vending machines, property boundaries, and the decoratively cut vegetation stretches. Typically where the urbanization level is high, subjects have a choice of a number of visual elements. 
Highly urbanized residential streetscapes are normally characterized by diverse elements for human requirements; therefore, to cater to their day-to-day requirements, retail shops, vending machines and some other business places like laundries, and saloons spring up among the houses. Thus, the variation of the streetscape increases and the subjects have a choice of a variety of elements to choose. Therefore, at the analysis stage of the perspective views, the subjects selected up to five visual elements from each viewpoint along the high density streetscapes. However, from urbanization controlled streetscapes, the selections were fewer and, in some viewpoints, there were no major visual elements for further classification. Visual perception differs from person to person; therefore, a single person's perception of figures and backgrounds will not be adequate for reliable and accurate analysis. For that reason, in this study, the perceptions of 20 people were used to identify the figures and the backgrounds. The probability of identifying an object as a figure or a background by 20 persons was used in differentiating the available visual elements into figures and backgrounds.

One more important point is that the selected major visual elements in highly urbanized streetscapes can possibly be further classified into a large number of figures and backgrounds. Since highly urbanized streetscapes associate with diverse human activities, subvisual elements used in the houses and small scale business places are varied. A visual element is a pool of figures and backgrounds (variety), which display a spatial hierarchy and varied spatial connections (dependency). If an element has lots of figures and backgrounds and a number of spatial connections, that element becomes prominent in the view. Therefore, the subjects could easily select such elements from the streetscape view and identify many subfigures and backgrounds. To find the variety and the dependency available within a major visual element, taxonomic diagrams have been utilized. The taxonomic diagram of major visual elements, with a large number of subvisual elements and spatial connections, becomes large in both vertical and horizontal directions. Consequently, the taxonomic diagram drawn for major visual elements in highly urbanized streetscapes was extended up to six levels and wide with more than 10 branches. In contrast, the taxonomic diagram of the urbanization controlled streetscapes had few levels and fewer than five branches. Urbanization controlled streetscapes had fewer human activities, but other activities like home gardening, paddy cultivation, and parking lots largely could be seen. Figures 6 and 7 depict the taxonomic diagrams drawn for a major visual element of views of urbanization controlled and highly urbanized residential streetscape. Bold labels refer to the figures in the taxonomy.

By just looking at the taxonomic diagrams of urbanization controlled and medium and highly urbanized streetscapes, it is possible to get an idea of the variety and dependency of the visual elements that contribute to the variations in structural hierarchical visual complexity of the streetscapes.

But what is additionally required is a concrete method to measure the visual complexity objectively. To meet this requirement, taxonomic entropy was utilized. Taxonomic entropy gives a numerical value to the structural hierarchical visual complexity, based on the length and the width of the taxonomic diagram. When the taxonomic diagram has a large number of branches and levels, the taxonomic entropy becomes high and vice versa.

Taxonomic entropy is a new approach for landscape and urban planning. The present research is the first attempt to use taxonomic entropy in measuring the structural hierarchical visual complexity of streetscapes. Besides, utilizing taxonomic diagram is a novel approach to show the variety and the dependency of visual elements.

After identifying the subvisual elements of a major visual element in urbanization controlled and medium and highly urbanized perspective views, they were arranged in terms of their taxonomic diagrams. This is followed by calculation of the taxonomic entropy, separately for each taxonomic diagram. Consequently, for highly urbanized streetscapes, a maximum of five taxonomic diagrams were drawn and as many taxonomic entropy values obtained for each viewpoint. Accordingly, for one highly urbanized streetscape, 25 taxonomic diagrams were drawn for 5 viewpoints and 25 taxonomic entropy values obtained. The average of the 25 values was taken as the final taxonomic entropy for the whole street. Similar calculations were made for the forward and backward directions of the all streetscapes.

The forward and backward taxonomic entropy values, twenty for each streetscape type, thus obtained for the urbanization controlled and medium and highly urbanized streetscapes are shown in Figures 8 and 9.

The taxonomic entropy is an indication of the structural hierarchical visual complexity of the streetscape. The forward taxonomic entropy ranged between 2.36 (lower entropy value) and 2.61 (upper entropy value) and mean value was 2.49 and the standard deviation was 0.08 , for urbanization controlled streetscapes, it ranged between 2.59 and 2.82 and mean value was 2.72 and the standard deviation was 0.07 , for medium urbanized streetscapes, and it ranged between and 2.79 and 2.98 and mean value was 2.89 and the standard deviation was 0.06 , for highly urbanized streetscapes. Principally, the forward streetscape taxonomic entropy ranged between 2.3 and 2.6 for urbanization controlled streetscapes, 2.6 and 2.8 for medium urbanized streetscapes, and 2.8 and 3.0 for highly urbanized streetscapes.

According to the calculations, the backward taxonomic entropy values ranged between 2.36 and 2.62 and mean value was 2.48 and the standard deviation was 0.07 , for urbanization controlled streetscapes, they ranged between 2.6 and 2.83 and mean value was 2.74 and the standard deviation was 0.07 , for medium urbanized streetscapes, and they ranged between 2.75 and 2.97 and mean value was 2.87 and the standard deviation was 0.06 , for highly urbanized streetscapes. Over all, the range of backward direction taxonomic entropy values is comparable with that of the forward direction entropy values.

As shown in Figures 8 and 9, it can be seen that the ranges of forward and backward entropy values are comparable except for minor variations in some streetscapes in both directions. The entropy values displayed significant differences with the building density of the streetscapes.

To statistically test the differences of taxonomic entropy values for forward and backward directions and the different 


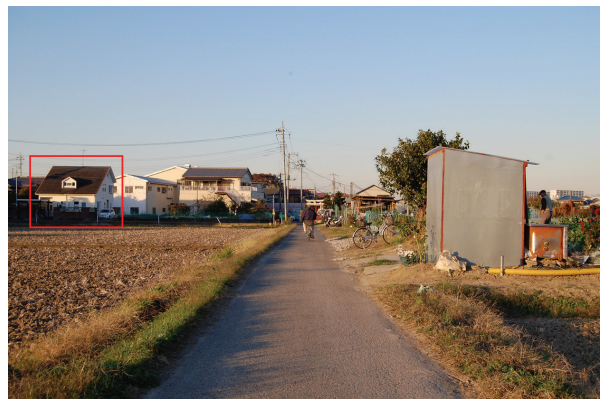

(a) Selected main visual element

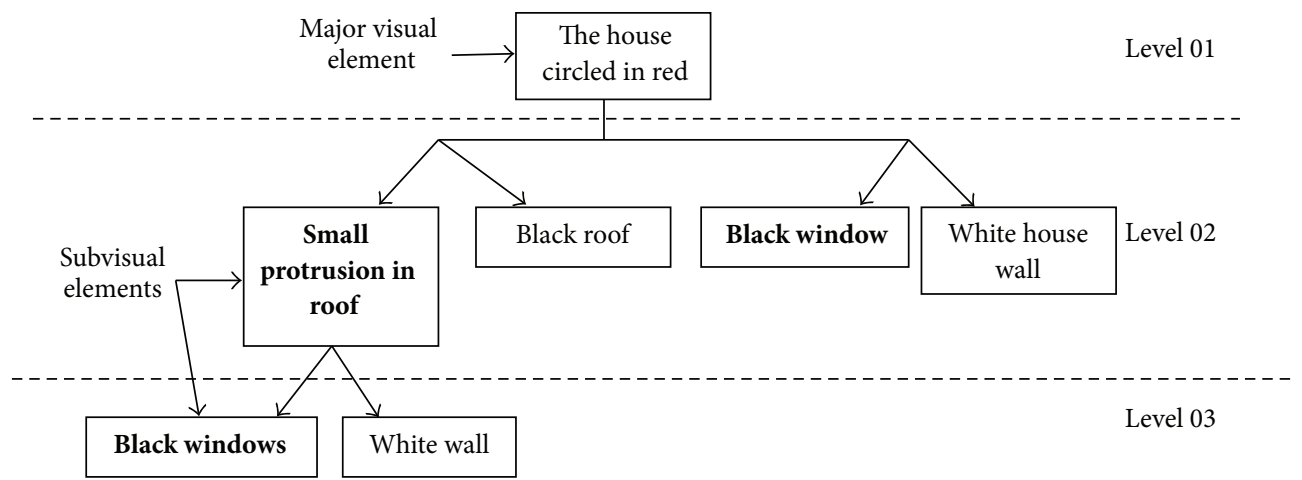

(b) Taxonomic diagram drawn to the main visual element

FIGURE 6: A perspective view of an urbanization controlled streetscape and the taxonomic diagram drawn for one of its major visual elements.

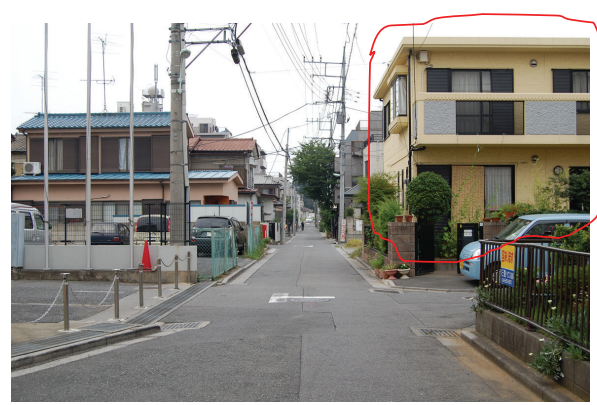

(a) Selected main visual element

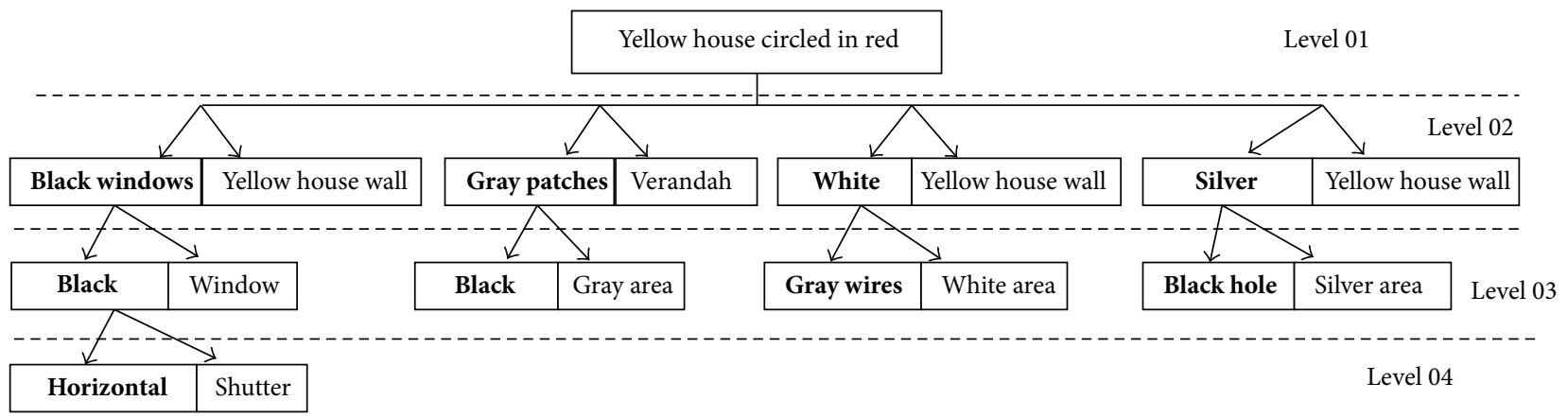

(b) Taxonomic diagram drawn to the main visual element

FigURE 7: A perspective view of a highly urbanized streetscape and a section of the taxonomic diagram of one of its major visual elements. 
TABLE 2: The results of the ANOVA test.

\begin{tabular}{|c|c|c|c|c|c|c|}
\hline Source of variation & SS & DF & MS & $F$ & $P$ value & $F$ crit \\
\hline Forward and backward & 0.000462 & 2 & 0.000231 & 0.051661 & 0.950 & 3.078057 \\
\hline Building density & 3.255095 & 2 & 1.627547 & 364.02 & 0.000 & 3.078057 \\
\hline Interaction & 0.020311 & 4 & 0.005078 & 1.135711 & 0.343 & 2.453458 \\
\hline Within & 0.496285 & 111 & 0.004471 & & & \\
\hline Total & 3.772153 & 119 & & & & \\
\hline
\end{tabular}

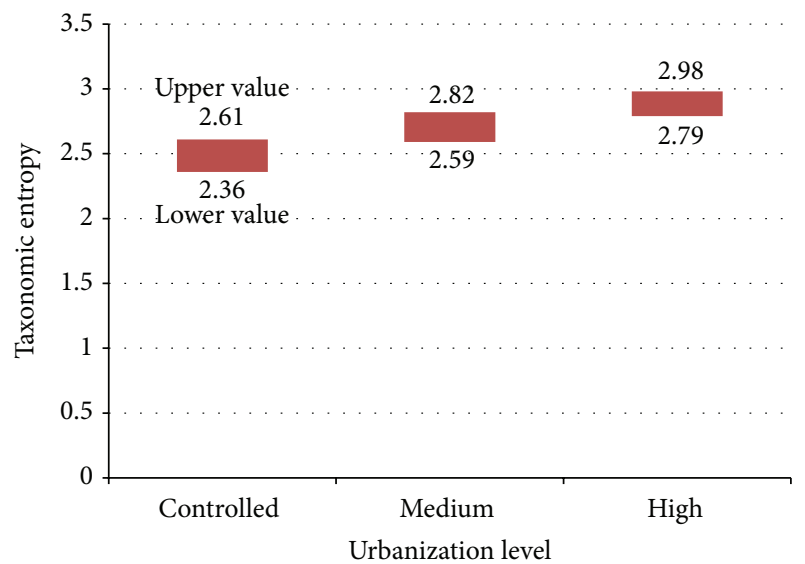

FIgURE 8: Taxonomic entropy values of urbanization controlled and medium and highly urbanized streetscapes in forward direction.

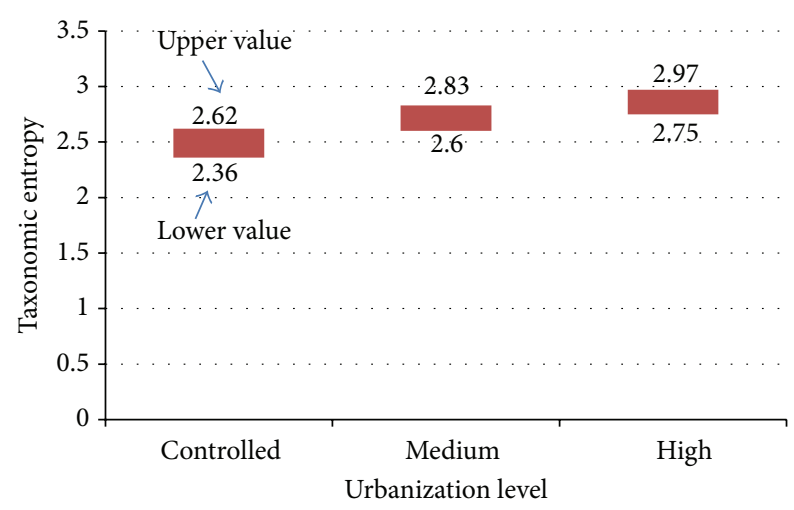

FIGURE 9: Taxonomic entropy values of urbanization controlled and medium and highly urbanized streetscapes in backward direction.

urbanization levels in the streetscapes, an ANOVA test was undertaken. The results of the ANOVA test are given in the Table 2.

The results displayed that there is no significant difference in the taxonomic entropy values for forward and backward directions of the streetscapes. The $P$ value for building density is less than $0.05(0.000)$; consequently, there is a significant difference of the taxonomic entropy based on the urbanization level of streetscapes.

Consequently, it can be concluded that the tested residential streetscapes of Saitama city display taxonomic entropy between 2.3 and 3.0 and there is no significant variation of the visual complexity in the forward and backward directions. However, the structural hierarchical visual complexity changes with the urbanization level near the street line. Figure 10 displays two perspective views with the lowest taxonomic entropy and the highest taxonomic entropy.

Urban planning area can be classified into urbanization area and the urbanization controlled area. The streetscapes in high building density areas can be considered as an urbanization area. Urbanization area associates with abundance of other visual elements like vegetation, boundaries of the properties, adornments on the buildings, and color variations. Therefore, more buildings involve more visual elements. Therefore, in urbanization areas the taxonomic entropy became high. The low density streetscapes in urbanization controlled area showed the lowest taxonomic entropy. These streetscapes are monotonous and they have very small amount of urban elements; therefore, the taxonomic entropy is low.

In general, from the tested streetscapes, the streetscapes in the urbanization areas displayed high taxonomic entropy values while the streetscapes in the urbanization controlled areas displayed lower taxonomic entropy values.

From the outcome of this research, it can be concluded that based on the availability of landscape features along the streetscapes, the taxonomic entropy differs. Higher urbanization means higher number of figures and backgrounds and their connections. Therefore, the structural hierarchical visual complexity of the streetscapes in the urbanization areas increases. Urbanization controlled areas have lower number of attractive figures and backgrounds and their connections; therefore, the structural hierarchical visual complexity becomes lower.

\section{Conclusions}

This research was undertaken to measure the structural hierarchical visual complexity of residential streetscapes. To achieve this objective, two approaches were used: (i) the figure and background classification of visual elements and (ii) taxonomic entropy analysis.

Figure and background classification technique was applied to find out the distinction and the connections among the visual elements of the streetscapes. The structural hierarchical visual complexity increases when the variety (distinction) and the dependency (connections) increase. Drawing taxonomic diagrams, to depict the identified distinctions and connections between the figures and backgrounds is 


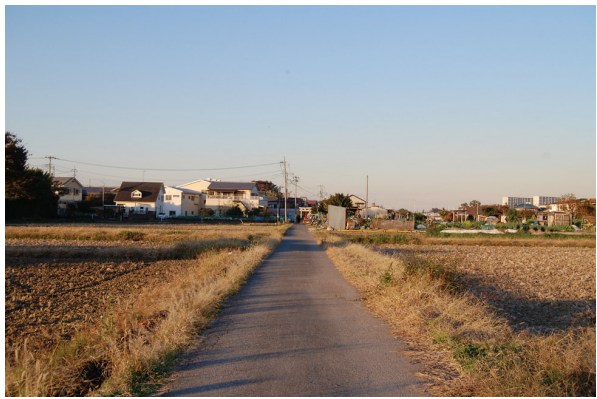

(a) Lowest taxonomic entropy

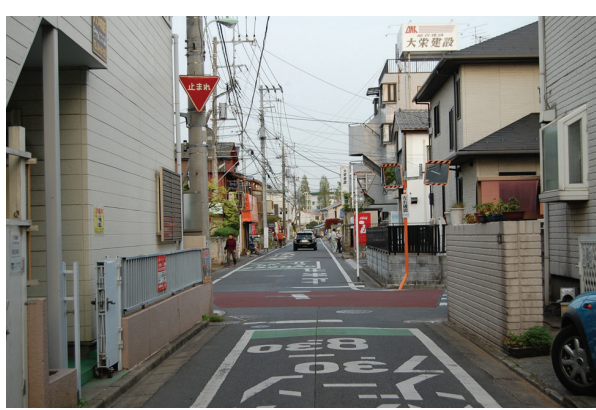

(b) Highest taxonomic entropy

FIGURE 10: Two perspective views of the (a) streetscape with lowest taxonomic entropy and the (b) streetscape with highest taxonomic entropy.

another approach applied in this research. As the variety and the connections among the visual elements in the streetscape increase and looking at the taxonomic diagrams of the streetscape elements, a clear idea emerges about the streetscape structural hierarchical visual complexity.

According to the calculations, 60 residential streetscapes located in the Saitama city, Japan, displayed taxonomic entropy between 2.3 and 3.0. These 60 residential streetscapes grouped into 20 streetscapes in highly urbanized areas, 20 streetscapes in medium urbanized areas, and 20 streetscapes in urbanization controlled areas based on the building density. Based on the urbanization level the taxonomic entropy differed. Consequently, the highly urbanized streetscapes obtained values between 2.8 and 3.0, medium urbanized streetscapes obtained 2.6 to 2.8 taxonomic entropy values, and the urbanization controlled residential streetscapes obtained values between 2.3 and 2.6.

Counting figures and backgrounds perceived by the observer is a difficult task. Visual perception varies from person to person; therefore, more observers and careful classification are needed for better classification of figures and backgrounds. This section was the most critical in this research and it consumed a lot of time. However, measurement of structural hierarchical visual complexity is more valuable than just structural visual complexity. Therefore, although the proposed method is time consuming, the result is worthwhile for the adaptation of this method in the future analysis.

Taxonomic entropy calculation primarily is based on the subjective analysis of figures and backgrounds. Therefore, the value may change based on the variation in human perception and based on the counting method of figures and backgrounds.

Taxonomic entropy represents the visual complexity level of streetscapes. It depends on the perceivable figures and backgrounds. Hence, this value is very useful for urban planners and architectural planners in designing streetscapes and building facades.

\section{Conflict of Interests}

The authors declare that there is no conflict of interests regarding the publication of this paper.

\section{References}

[1] J. H. Elder and R. M. Goldberg, "Ecological statistics of Gestalt laws for the perceptual organization of contours," Journal of Vision, vol. 2, no. 4, pp. 324-353, 2002.

[2] Y. Elsheshtawy, "Urban complexity: toward the measurement of the physical complexity of street-scapes," Journal of Architectural and Planning Research, vol. 14, no. 4, pp. 301-316, 1997.

[3] R. Rosenholtz, Y. Li, and L. Nakano, "Measuring visual clutter," Journal of Vision, vol. 7, article 17, 22 pages, 2007.

[4] S. Kaplan, R. Kaplan, and J. S. Wendt, "Rated preference and complexity for natural and urban visual material," Perception \& Psychophysics, vol. 12, no. 4, pp. 354-356, 1972.

[5] G. Jahn, A. Oehme, J. F. Krems, and C. Gelau, "Peripheral detection as a workload measure in driving: effects of traffic complexity and route guidance system use in a driving study," Transportation Research Part F: Traffic Psychology and Behaviour, vol. 8, no. 3, pp. 255-275, 2005.

[6] R. Kaplan, S. Kaplan, and R. Ryan, With People in Mind: Design and Management of Everyday Nature, Island Press, Washington, DC, USA, 1998.

[7] D. E. Berlyne, Studies in the New Experimental Aesthetics, Wiley, New York, NY, USA, 1974.

[8] B. B. Mandelbrot, The Fractal Geometry of Nature, W.H. Freeman and Company, New York, NY, USA, 1977.

[9] R. Näsänen, H. Kukkonen, and J. Rovamo, "Spatial integration of band-pass filtered patterns in noise," Vision Research, vol. 33, no. 7, pp. 903-911, 1993.

[10] F. W. Campbell and J. G. Robson, "Application of Fourier analysis to the visibility of gratings," Journal of Physiology, vol. 197, no. 3, pp. 551-566, 1968.

[11] R. E. Desrochers and M. Anand, "From traditional diversity indices to taxonomic diversity indices," International Journal of Ecology and Environmental Sciences, vol. 30, no. 2, pp. 85-92, 2004.

[12] L. N. Piotrowski and F. W. Campbell, "A demonstration of the visual importance and flexibility of spatial-frequency amplitude and phase," Perception, vol. 11, no. 3, pp. 337-346, 1982.

[13] V. Chikhman, V. Bondarko, M. Danilova, A. Goluzina, and Y. Shelepin, "Complexity of images: experimental and computational estimates compared," Perception, vol. 41, no. 6, pp. 631647, 2012

[14] F. Heylighen, "What is complexity? Principia Cybernetica Web," 1996, http://pespmcl.vub.ac.be/complexi.html. 
[15] D. C. Donderi, "An information theory analysis of visual complexity and dissimilarity," Perception, vol. 35 , no. 6, pp. 823835, 2006.

[16] A. Cavalcante, A. Mansouri, L. Kacha et al., "Measuring streetscape complexity based on the statistics of local contrast and spatial frequency," PLOS ONE, vol. 9, no. 2, Article ID e87097, 2014.

[17] A. Forsythe, N. Sheehy, and M. Sawey, "Measuring icon complexity: an automated analysis," Behavior Research Methods, Instruments, \& Computers, vol. 35, no. 2, pp. 334-342, 2003.

[18] J. Cooper, "Fractal assessment of street-level skylines: a possible means of assessing and comparing character," Urban Morphology, vol. 7, no. 2, pp. 73-82, 2003.

[19] R. M. Warwick and K. R. Clarke, “New 'biodiversity' measures reveal a decrease in taxonomic distinctness with increasing stress," Marine Ecology Progress Series, vol. 129, no. 1-3, pp. 301305, 1995.

[20] R. Rosenholtz, Y. Li, J. Mansfield, and Z. Jin, "Feature congestion: a measure of display clutter," in Proceedings of the SIGCHI Conference on Human Factors in Computing Systems (CHI '05), pp. 761-770, ACM, Portland, Ore, USA, April 2005.

[21] C. Ricotta and G. C. Avena, "An information-theoretical measure of taxonomic diversity," Acta Biotheoretica, vol. 51, no. 1, pp. 35-41, 2003. 

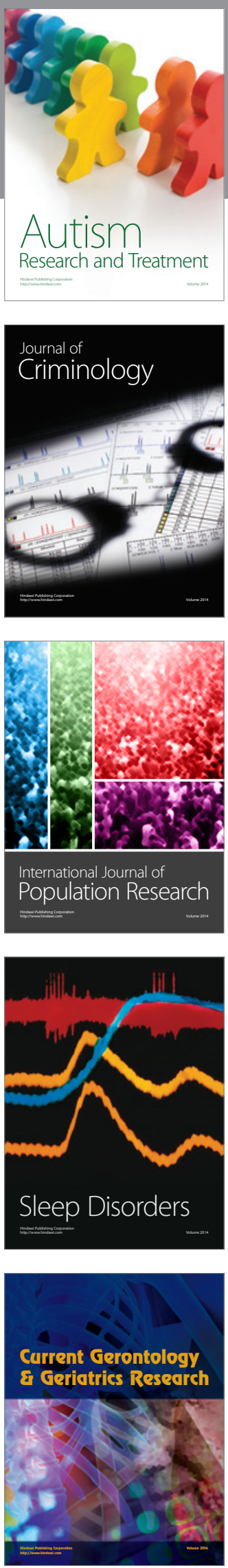
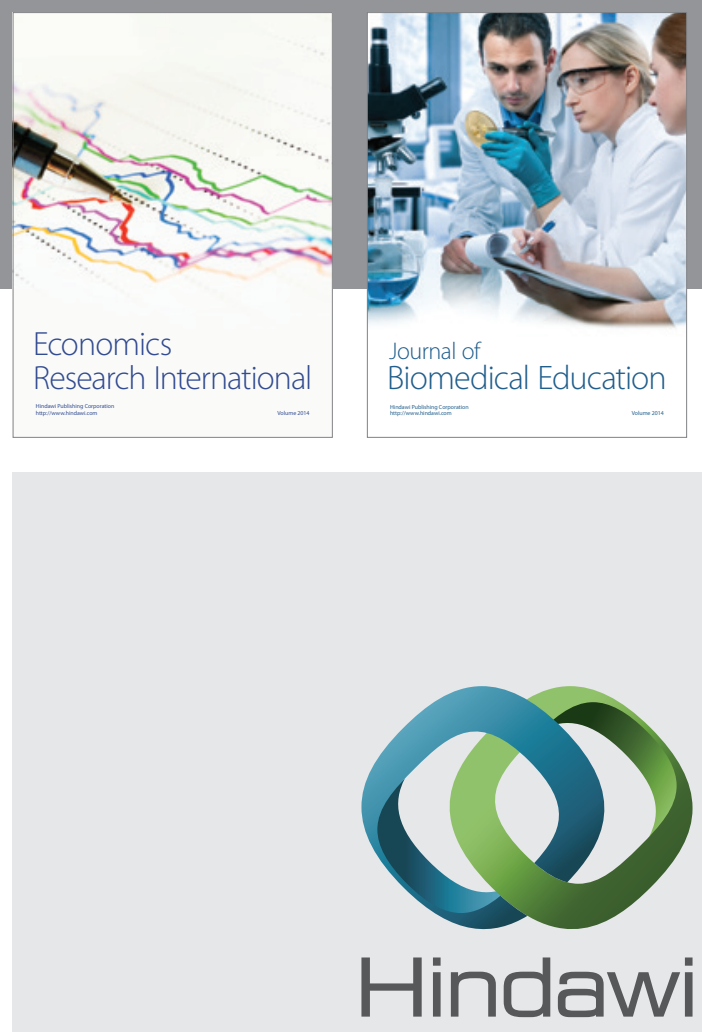

Submit your manuscripts at

http://www.hindawi.com
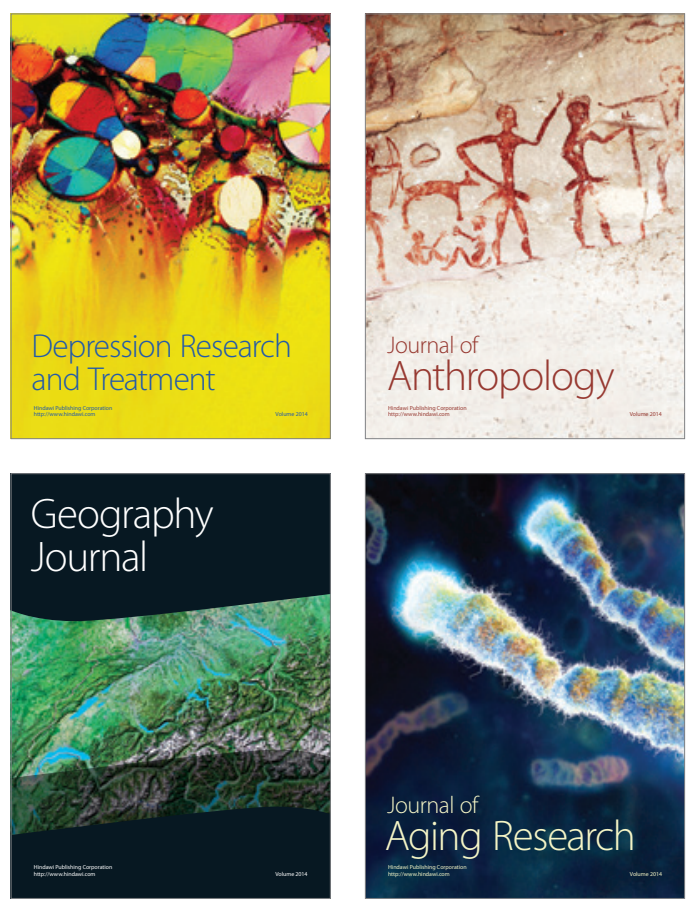
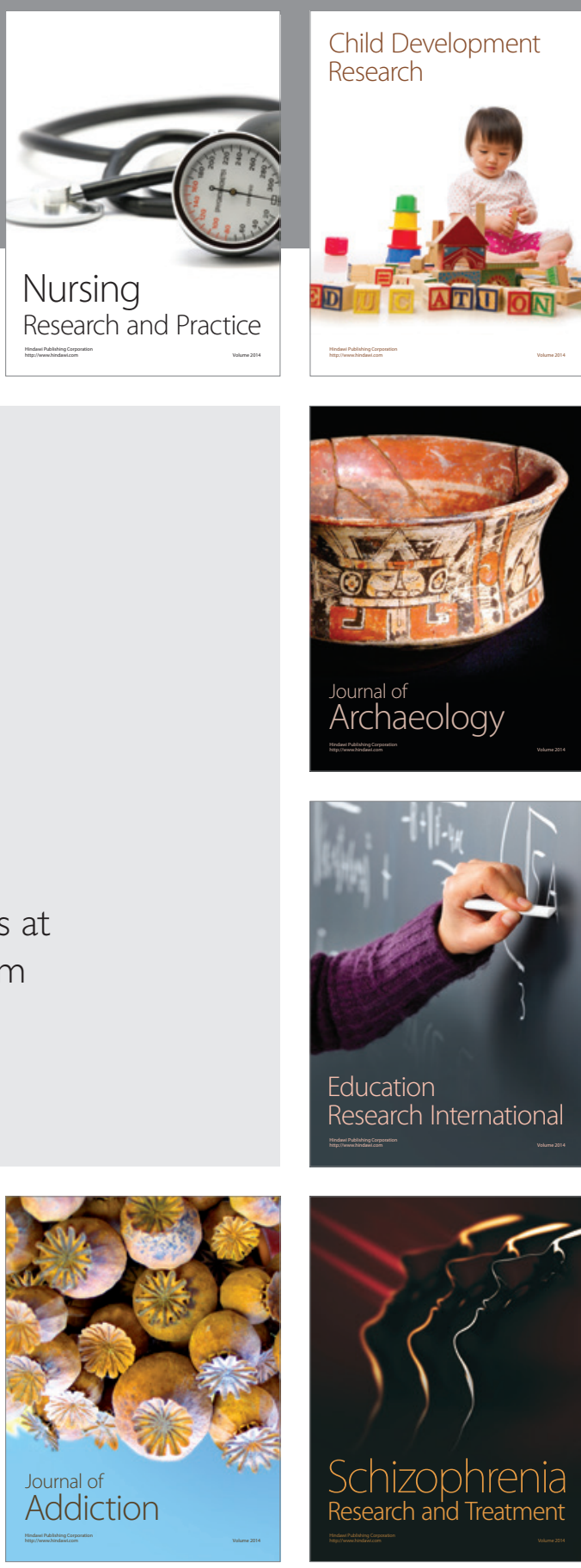

(D)
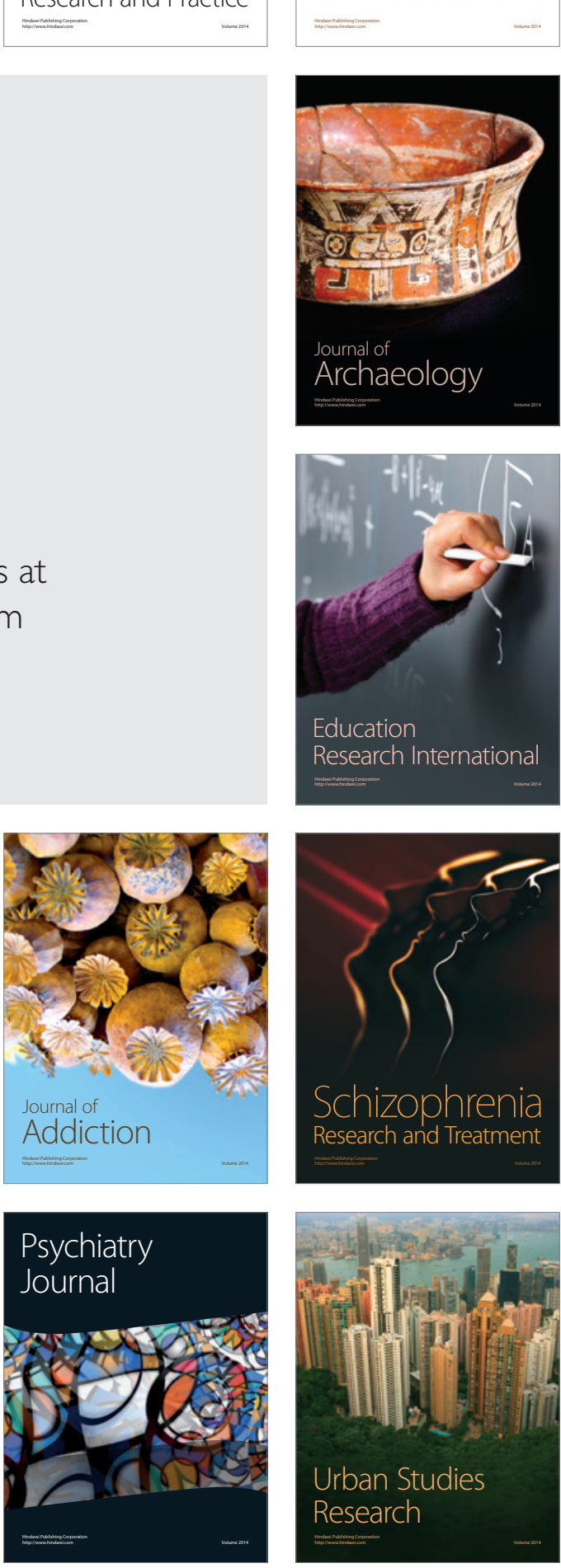